\title{
CODING OF SPECTRALLY HOMOGENEOUS REGIONS IN MULTISPECTRAL IMAGE COMPRESSION
}

Gabriel Fernàndez

Signal Processing Laboratory

Swiss Federal Institute of Technology

CH-1015 Lausanne, Switzerland

E-mail: fernandez@lts.epfl.ch

\section{ABSTRACT}

In this paper we present a new approach in the compression of multispectral images. It is based on the merging of two main tendencies such as the use of KLT as a spectral decorrelator and object based image coding schemes. The use of the principal component in multispectral imagery is described and used to perform a multispectral segmentation. This segmentation is taken as the basis for a specific spectral decorrelation for each segmented class. The resulting eigen images present lower variance than classical KLT approaches. Each of the eigen regions is coded spatially using a shape adaptive DCT algorithm. The method outperforms non-region multispectral KLT+DCT schemes as well as JPEG, while adding the region based functionalities.

\section{INTRODUCTION}

Multispectral image compression has for many years been a topic of interest to image processing and remote sensing researchers. From the image processing point of view it is a challenging field, where one faces trade-offs between data size, coding complexity, and reconstruction fidelity. The reason that multispectral image compression provides a different solution than standard still image compression is the possibility of exploiting the spectral correlation.

Many efforts have been made in the compression of multispectral images. Algorithms have been applied for lossless[1], near-lossless and lossy applications. Most of them use the spectral correlation between spectral bands in order to achieve better compression ratios. Among others, classical algorithms are based on Vector Quantization[2], 3dimensional transformations, spectral prediction[3], wavelets or hybrid methods such as DPCM-DCT[4] or KLTDCT[5]. The use of KLT (Karhunen-Loève Transform also known as Hotelling or Principal Component) as spectral decorrelator has been shown to be one of the most effective[6].

On the other hand, in still and video coding fields, the introduction of region based methods $[7,8]$ has been an important new development. These coding schemes' main advantage is the use of arbitrarily shaped regions of support allowing better adapted transformations in the spatial domain. The region based coding yields reconstructed images with artifacts that are less bothersome to the Human
Craig M. Wittenbrink

\author{
Computer Engineering \& Information Sciences \\ University of California, Santa Cruz \\ Santa Cruz, CA 95064 \\ E-mail: craig@cse.ucsc.edu
}

Visual System than block based artifacts. Another advantage of region based methods is object scalability, where objects are coded according to different criteria.

No work has been reported in the merging of these two fields. This is the goal of this paper, we present a region based method for multispectral images. Where spectral decorrelation is done internally for each spectrally homogeneous region and the spatial coding takes advantage of the existing techniques of object based coding. The paper is presented in three main parts: $i$ ) a first part devoted to the analysis of multispectral datasets in order to determine the spectral homogeneous classes, ii) the spectral decorrelation applied to each class and iii) the exploitation of the spatial correlation by coding the inside of each class.

\section{MULTISPECTRAL SEGMENTATION}

\subsection{KLT and multispectral imagery}

One very successful application of the KL transformation is in multispectral images. The transformation is applied in the spectral dimension taking each one of the pixels of the scene as $N$ dimensional vectors $N$ being the number of spectral bands. Let $X$ be the vector containing the $N$ components for a given pixel and $U$ the mean vector $U=$ $E[X]$. The covariance matrix $C_{x}$ is defined as:

$$
C_{x}=E\left[(X-U)(X-U)^{t}\right]
$$

The Karhunen-Loève Transfomation $(T)$ is defined as the one that diagonalizes $C_{x}$ in the following way:

$$
C_{y}=T C_{x} T^{t}=\Lambda
$$

where $C_{y}$ is the covariance of the transformed vector $(Y)$ and $\Lambda$ the diagonal matrix representing eigenvalues. $Y$ can then be obtained by the equation:

$$
Y=T(X-U)
$$

Since the transformation optimally diagonalizes the covariance matrix between spectral bands, the spectral correlation of the transformed components is removed.

The images in the transformed domain are sorted in order of importance or with decreasing variance (value of the eigenvalues). This energy compaction in the spectral axis is quite suitable for selection of the main spectral components for analysis as well as for image compression. 


\subsection{Multispectral segmentation}

Given a multispectral dataset the KLT is applied across the spectral dimension. From the obtained main components, one selects the most important. Experimental results show that selecting the first three main components is a good trade-off between complexity of the subsequent analysis and spectral information retrieval. Of course, this may vary with the nature of the scene. The advantage of using KLT is twofold in the following sense: it reduces the problem of band selection for the classification to a simple choice of the total number of images. And, it assures that the selected images for classification are the most representatives of the spectral content in the scene.

Different clustering techniques can be applied for segmentation. We have chosen the multidimensional histograming of the three main components because it does not require any recursive calculation that would slow down the system. Once the 3-dimensional histogram is built (a scaled version due to storage constrains), some morphological operations are applied to the four dimensional surface that represents the histogram, these are: smoothing and peak detection by morphological opening operators. Maximal peaks are extracted, sorted in order of importance and selected depending on the number of required classes. All the pixels are classified following the criterion of maximum correlation and a segmentation map is generated. This segmentation map is cleaned by an opening by reconstruction morphological filter [9] in order to remove small sized regions and avoiding their inefficient coding. An application of this segmentation has been successfully applied to cloud extraction for a $3 \mathrm{D}$ cloud visualization[10].

\section{SPECTRAL DECORRELATION}

\subsection{Classical spectral decorrelation approaches}

The KLT applied to multispectral images for image compression has been used in several approaches. The basic approach is the one that takes all the spectral images and computes the KLT in the spectral axis. Since the resulting components are sorted in order of importance they can be easily quantized with criteria that take into account the importance factor, achieving high compression ratios. Better results have been obtained when the images are partitioned in regular blocks and the KLT is computed within these blocks[11]. The drawback of the latter approach is the high overhead information needed when the number of bands increases (images can contain hundreds of blocks and hundreds of bands).

\subsection{Region based spectral decorrelation}

We introduce a new approach to the application of KLT for multispectral image compression. Taking the multispectral segmentation described in section 2.2 , a different transformation is calculated for each class in the following way:

$$
C_{y i}=T_{i} C_{x i} T_{i}^{t}=\Lambda_{i}
$$

where $i$ is the class number and $C_{x i}$ is the covariance matrix calculated from the pixels belonging to the class $i$.
Since the obtained segmentation is the result of the classification of the principal components, all the pixels of the same class are likely to have a similar KLT transformation, thus the transformation is going to be better adapted to statistics of the set. If we apply a different KLT to each class, the energy is going to be optimally compacted for each region, giving highly energy compacted eigen images with less variance than the obtained by a simple KLT. Since a different transformation is computed for each class (not objects) the overhead data volume does not increase substantially as discussed in the following subsection. Figure 1 illustrates the region based KLT approach.

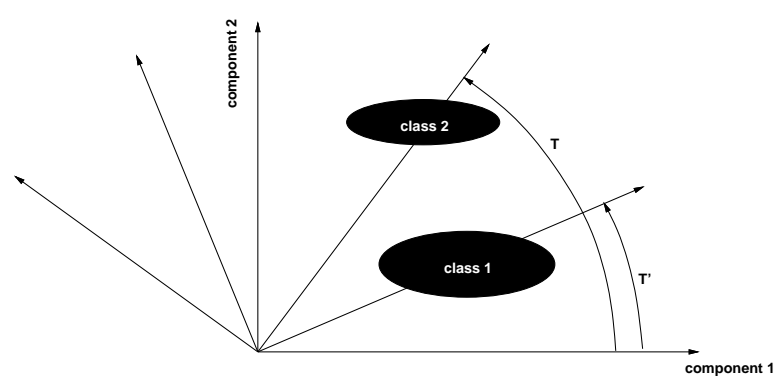

Figure 1: Example of two different KL transformations applied to two spectral clusters.

\subsection{Overhead information}

The overhead information is all data needed for the decoder not directly related to the pixel values. In a KLT based algorithm this information is reduced to the covariance $C_{X}$ matrix and the mean vector $U$ of eq. 1 . The volume of this overhead will depend on the number of bands and the number of transformations needed in the algorithm (i.e. the number of blocks in a block based scheme or classes in our approach). Note that in the presented region based transform, a different KLT is applied to each class and not to each single region. That means that in a satellite image we define a KLT for the class of clouds, one for the class of land, etc... and not for every single cloud. Since all the clouds have similar spectral signatures, the KLT is always optimal but the overhead information to encode does not substantially increase.

In the presented algorithm, a second overhead component is the coding of the segmentation information. We have to point out that this overhead information can be highly compressed (few classes are usually defined), and that its cost is divided by the number of bands to compress leading to minimal overhead in the case of hyperspectral images.

Figure 2 shows the evolution of the overhead information as the number of bands to code increases. Values are plotted for a block based KLT (64*64 block size) and for 3 and 6 region KLTs. Note that for a given number of bands, the overhead of the block based scheme is largely above the region based ones even if the latter ones include the segmentation cost. 


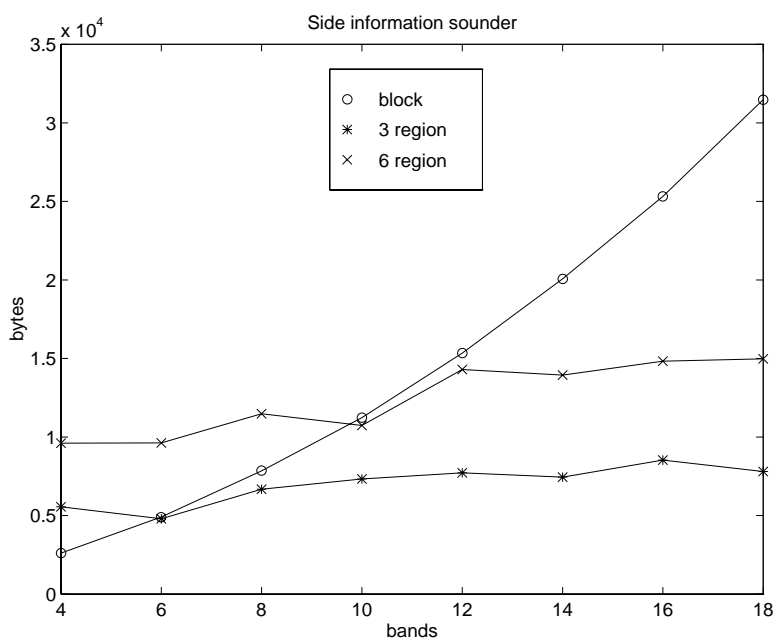

Figure 2: Overhead information when increasing the number of spectral bands.

\section{REGION CODING}

\subsection{Spatial coding approaches}

After the spectral decorrelation, all the spatial correlation existing in the principal components remains to be exploited. Several approaches can be observed at this point and they are related to classical still image coding ( DCT, subband coding, ...). Regions can be coded by shape adaptive methods based on subband coding or DCT $[12,13,8]$. Furthermore, the segmented regions belonging to the same sort of terrain or clouds are likely to have homogeneous textures, thus texture based coding methods can also be applied. Simpler approaches can be DPCM inside the regions. Two of these possible approaches have been adopted in this paper, and they are explained in the following subsections.

\subsection{Runlength of regions}

A simple spatial coding of the components has been adopted in a first step, images are quantized differently depending on its importance factor and a DPCM followed by run-length coding is applied. Finally, the resulting bitstream is entropy coded.

\subsection{Region based DCT}

The second spatial coding we use is the very well known DCT. This spatial transformation is widely used in many coding schemes for still images and video. In multispectral images has also been frequently applied[11]. Most of these methods use a block based partition of the image in order to perform the DCT. In the new tendency of the use of region based methods, a shape adaptive DCT has been developed by [13]. This low complexity implementation of the shape-adaptive DCT is able to code arbitrarily shaped regions independently one to each other. Once the shape
DCT coefficients are obtained, the following processing is the classical: quantization, zig-zag scanning and entropy coding. The quantization tables applied are different depending on the number of KL component.

\section{RESULTS}

The presented method was applied to multispectral images of the imager and sounder instruments of GOES- 8 NOAA satellite. These datasets contain 5 and 19 spectral bands respectively. The variance reduction in the main component when the region based KLT is applied is more than $70 \%$ with respect to the normal KLT and $30-50 \%$ when compared with the block based KLT $(64 * 64$ block size $)$ as listed in table 1 . The algorithm quantizes the eigenimages differently (using a given quality factor). The two spatial coding approaches described in section 4 have been applied to the spectrally decorrelated components. Results are shown in figure 3 and compared to classical KLT and to the standard JPEG when coding the bands separately. As expected, DCT based methods outperform the simple runlength. The proposed algorithm has a gain of $0-2 \mathrm{~dB}$ over non-region based KLT algorithms depending on the number of bands and compression rate.

\begin{tabular}{|c|c|c|c|}
\hline num. bands & KLT type & Var comp.1 & reduction \\
\hline \hline \multirow{2}{*}{$\begin{array}{c}\text { sounder- } \\
19 \text { banbs }\end{array}$} & classical & 14677.76 & \\
\cline { 2 - 4 } & block & 6122.71 & $58.28 \%$ \\
\cline { 2 - 4 } & 3 regions & 4469.41 & $69.54 \%$ \\
\hline \hline \multirow{2}{*}{$\begin{array}{c}\text { imager- } \\
5\end{array}$ banbs } & classical & 7892.67 & \\
\cline { 2 - 4 } & block & 4427.88 & $43.8 \%$ \\
\cline { 2 - 4 } & 3 regions & 2180.92 & $72.36 \%$ \\
\hline
\end{tabular}

Table 1: Variance reduction of the main component for the different KLT spectral decorrelators

The performance of the proposed algorithm has to be evaluated not only in rate/distortion terms but also in the terms of functionalities and visual quality. For instance, the region based approach did not present annoying block artifacts. Furthermore, one of the main advantages of using object based coding methods is the object scalability, where regions can be compressed separately, or compressed by different methods depending on some user priorities. For example, meteorologists specialized on cloud analysis may be interested in compressing the multispectral information related only to clouds and neglect other region information by coding it with fewer bits or even not coding it.

\section{CONCLUSIONS}

In this paper we have presented a new approach on the coding of multispectral images. It is based on the merging of two tendencies in image coding and remote sensing coding. The classical spectral decorrelator such as KLT is combined with region based coding techniques, obtaining the so-called region based KLT. The spatial coding of the spectrally decorrelated regions is done by shape adaptive 

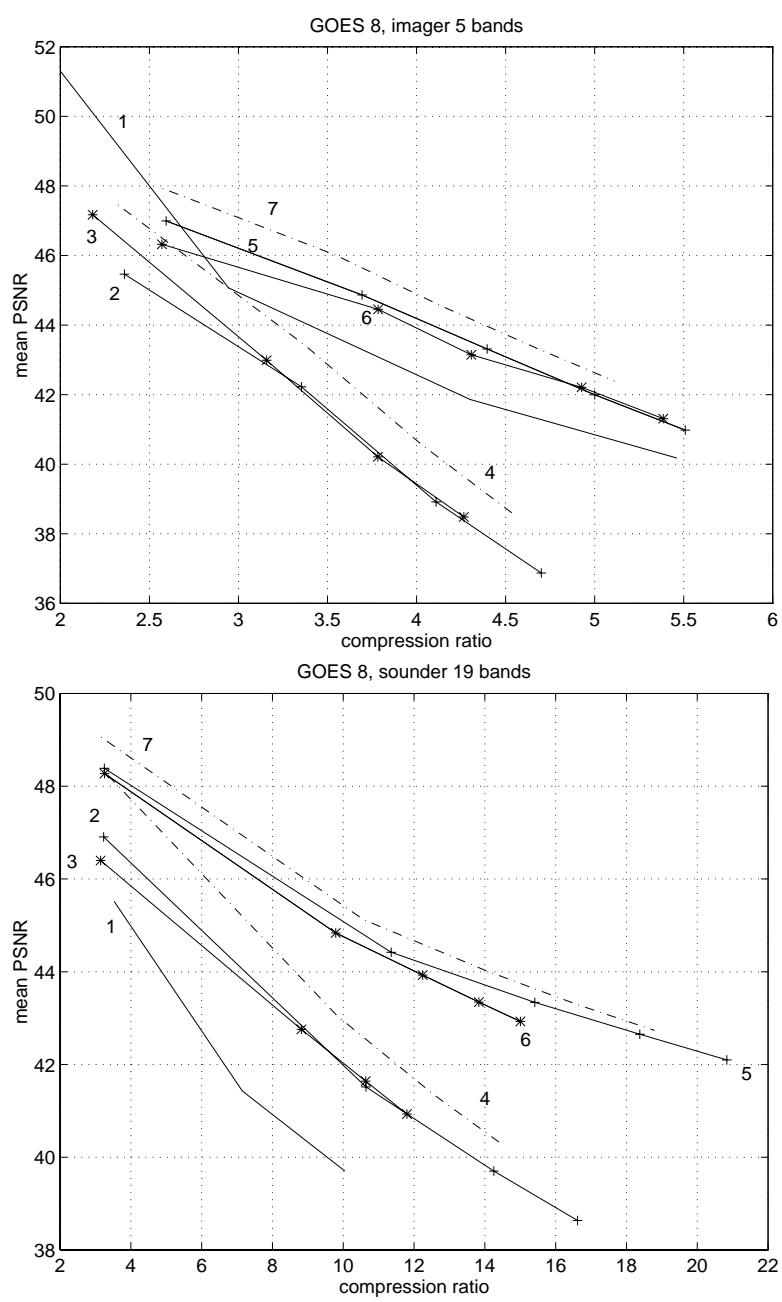

Figure 3: Rate distortion curves for imager (top) and sounder (bottom) instruments. 1 JPEG, 2. KLT + DPCM, 3. block KLT + DPCM, 4. 3-region KLT+ DPCM, 5. KLT + DCT, 6. block KLT + DCT, 7. 3-region KLT + SADCT

DCT. The algorithm outperforms differently the classical KLT+DCT multispectral schemes depending on the number of bands, nature of the scene and quality of the segmentation. Furthermore, the method has the advantage of embedding a segmentation map and the possibility to code each region differently.

\section{Acknowledgments}

The authors of this paper would like to thank Prof. Glen G. Langdon and Prof. Murat Kunt for making this work possible. Images were provided by the NASA-Goddard Space Flight Center, and the shape adaptive DCT source code by the Heinrich-Hertz-Institut fuer Nachrichtentechnik, Berlin.

\section{REFERENCES}

[1] Nasir D. Memon, Khalid Sayood, and Spyros S. Magliveiras. Losless compression of multispectral image data. IEEE Transcations on Geoscience and Remote Sensing, 32(2):282-289, March 1994.

[2] Smita Gupta and Allen Gersho. Feature predictive vector quantization of multispectral images. IEEE Transactions on Geoscience and Remote Sensing 30(3):491-501, May 1992.

[3] Ashok K. Rao and Sanjai Bhargava. Multi-spectral data compression using interband prediction. In Industry Workshop, Image Compression - Applications and Innovations, Snowbird, Utah, April 1994.

[4] Glen P. Abousleman, Michael W. Marcellin, and Bobby R. Hunt. Compression of hyperspectral imagery using the 3-d dct and hybrid dpcm/dct. IEEE Transactions on Geoscience and Remote Sensing, 33(1):2634, January 1995.

[5] John A. Saghri, Andrew G. Tescher, and John T. Reagan. Practical transform coding of multispectral imagery. IEEE Signal Processing Magazine, pages 32-43, January 1995.

[6] Patrick J. Ready and Paul A. Wintz. Information extraction, snr improvement, and data compression in multispectral imagery. IEEE Transactions on communications, COM-21(10):1123-1131, October 1973.

[7] H.G. Musmann, M. Hotter, and J. Ostermann. Objectoriented analysis-synthesis coding of moving images. Signal Processing: Image Communication, 1(2):117$138,1989$.

[8] S-F. Chang and D.G. Messerschmitt. Transform coding of arbitrary-shaped image segments. In Proc. 1st ACM Internat. Conf. on Multimedia, pages 83-90, Anaheim, CA, 1993.

[9] J. Serra. Image Analysis and Mathematical Morphology. Academic Press, Inc., Ecole Netionale Supérieure des Mines de Paris, Fontainebleau, 1982.

[10] Craig M. Wittenbrink, Gabriel Fernàndez, and Glen G. Langdon. Feature extraction of clouds from goes satellite data for integrated model measurement visualization. In Proceedings of SPIE/ISEST Conference, San Jose, February 1996.

[11] John A. Saghri and Andrew G. Tescher. Near-losless bandwidth compression for radiometric data. Optical Engineering, 30(7):934-939, July 1991.

[12] M. Gilge, T. Engelhardt, and R. Mehlan. Coding of arbitrarily shaped image segments based on a generalized orthogonal transform. Signal Processing: Image Communication, 1(2):153-180, 1989.

[13] Thomas Sikora. Low complexity shape-adaptive dct for coding of arbitrarily shaped image segments. Signal Processing: Image Communication, 7:381-395, 1995. 